\title{
Calcificación idiopática digital
}

\author{
I. Proubasta, C. Lamas, A. Millán. \\ Servicio de Cirugía Ortopédica y Traumatología. Hospital de la Santa Cruz y San Pablo. Barcelona. \\ Universidad Autónoma de Barcelona. España.
}

Resumen: Se presenta un caso de calcificación idiopática digital, también llamada peritendinitis o periartritis calcificante, entidad muy poco conocida que cursa con dolor e inflamación aguda y que simula una tenosinovitis de origen séptico y/o gotoso. No obstante, el estudio radiográfico permite confirmar el diagnóstico. Asimismo, a pesar de que su curso natural es hacia la resolución espontánea de los depósitos cálcicos, se recomienda la inmovilización y la administración de AINES y/o corticoides.

Palabras clave: Calcificación idiopática, periartritis, peritendinitis.

\begin{abstract}
We report a case of digital idiopathic calcification, also called calcific peritendinitis or periarthritis, a rare condition that causes severe pain and swelling and that simulating a septic origin or gout. However, the radiographic study confirm the diagnosis. Also, despite its natural course being towards spontaneous resolution of calcic deposits, it is recommended the immobilization and NSAIDs and/or corticoids administration.
\end{abstract}

Key words: Idiopathic calcification, periarthritis, peritendinitis.

La calcificación idiopática digital (CID), también conocida como peritendinitis o periartritis calcificante, constituye una entidad muy poco conocida a nivel de la mano y, a menudo, se la diagnostica erróneamente como una tenosinovitis gotosa y/o séptica'. Es por ello, que es preciso tener un diagnóstico cierto para evitar tratamientos innecesarios, especialmente la administración de antibióticos y/o el desbridamiento quirúrgico, pues en general la historia natural de estos depósitos cálcicos es hacia la desaparición espontánea en el curso de 2-3 semanas, aunque la infiltración con corticoides y/o la administración oral de AINES/corticoides acortan dicho periodo.

El objetivo de este trabajo es el de presentar un nuevo caso de CID y revisar la bibliografía sobre esta particular patología.

\section{CASO CLÍNICO}

Paciente mujer de 32 años de edad, sin antecedentes personales ni patológicos de interés, acude a la consulta por dolor agudo y limitación de la movilidad del dedo índice izquierdo de tres días de evolución. Niega antecedente traumático previo, aunque su trabajo requiere de movimientos repetitivos de los dedos. A la exploración se observa una inflamación eritematosa y generalizada del citado dedo con dolor a la presión en la articulación interfalángica proximal (IFP), más acusado en el lado radial. No existen déficits sensitivos y la movilidad articular se encuentra restrin- gida, pudiendo flexionar la IFP solo $30^{\circ}$. La analítica sanguínea es completamente normal, a excepción de un ligero aumento de la VSG $(22 \mathrm{~mm} / \mathrm{h})$. La paciente está afebril. El estudio radiográfico muestra la presencia de una calcificación situada en la zona radiopalmar de la articulación IFP @ Figura I. Con el diagnóstico de CID, se inmovilizó el dedo con una férula digital y se inició tratamiento médico con dexametasona oral (Fortecortin ${ }^{\circledR}$ comprimidos de Img), a dosis de I mg cada 8 horas durante tres días, seguidos de I mg cada 12 horas durante tres días más y, finalmente, Img al día otros tres días. Tras dicho tratamiento, fue visitada de nuevo constatando la ausencia de dolor e inflamación y con un arco de movilidad prácticamente normal. Al mes se repitió el estudio radiográfico, observando que había desaparecido la calcificación (1) Figura 2. Un año después del episodio agudo, no ha vuelto a tener ninguno más.

\section{DISCUSIÓN}

La CID a nivel de la mano, fue descrita por primera vez en 1924 por Cohen, específicamente a nivel del Flexor Carpi Ulnaris. En este contexto, dicha entidad es frecuente observarla a nivel del hombro pero en la

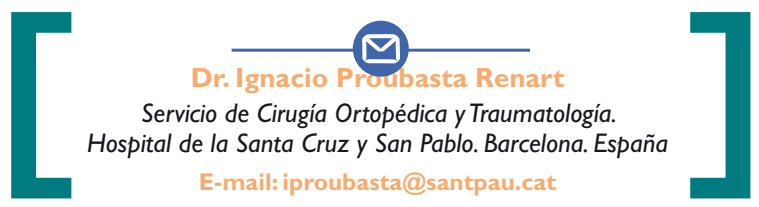




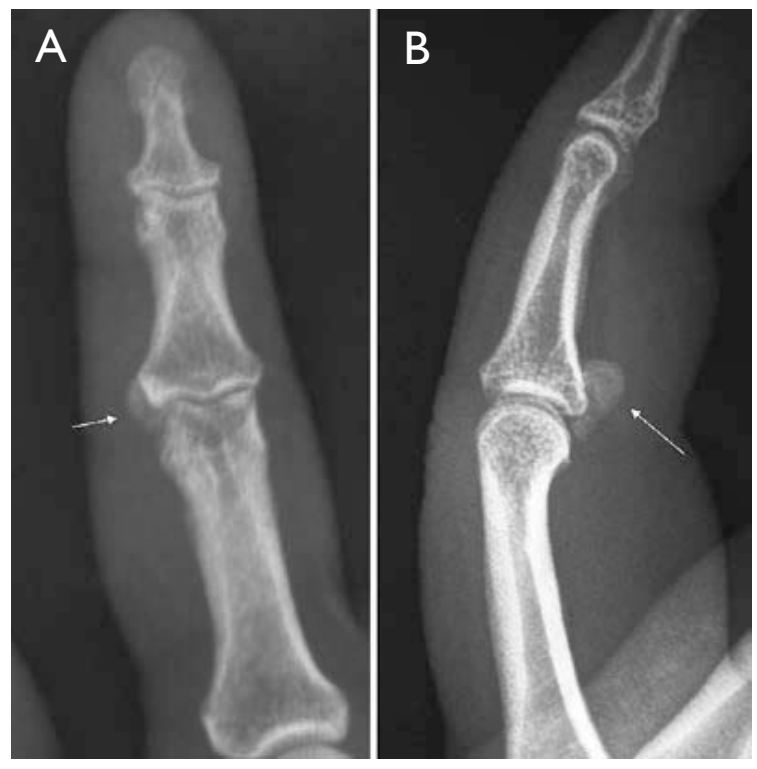

Figura I. Estudio radiográfico del dedo indice donde se observa la presencia de una calcificación periarticular a nivel de la interfalángica proximal. A. Proyección anteroposterior. B. Proyección de perfil.

muñeca y especialmente en dedos es inhabitual. Así, Sandstrom, en 1938, publicó una serie de 329 casos de peritendinitis calcificante, de las que el $79 \%$ afectaron la región del hombro mientras que el $2 \%$ lo hicieron a nivel de la mano. Sin embargo, las series publicadas por Yelton y Dickey en 1958 y Carroll y cols. en 1955, indican que dicha frecuencia es algo mayor, afectando más las articulaciones metacarpofalángicas ${ }^{2-9}$ que las interfalángicas. Dentro de estas últimas, la proximal es la que se observa más habitualmente, mientras que la distal sería una localización excepcional.

La etiología de estos depósitos cálcicos es desconocida. Algunos autores consideran que es el resultado de una hipoxia tisular local, mientras que otros creen que es la acción de un traumatismo (agudo y/o repetitivo). Sin embargo, todo parece indicar que es la combinación de ambos (hipoxia tisular más traumatismo) el verdadero causante del problema. En nuestra paciente, el hecho de trabajar en una cadena de montaje con movimientos repetitivos de los dedos, quizás fue un elemento favorecedor en la aparición de la calcificación. Sea como fuere, es importante resaltar que la sintomatología aguda que se produce es consecuencia de la ruptura del depósito cálcico que actúa como agente inflamatorio y no por la presencia del mismo5.
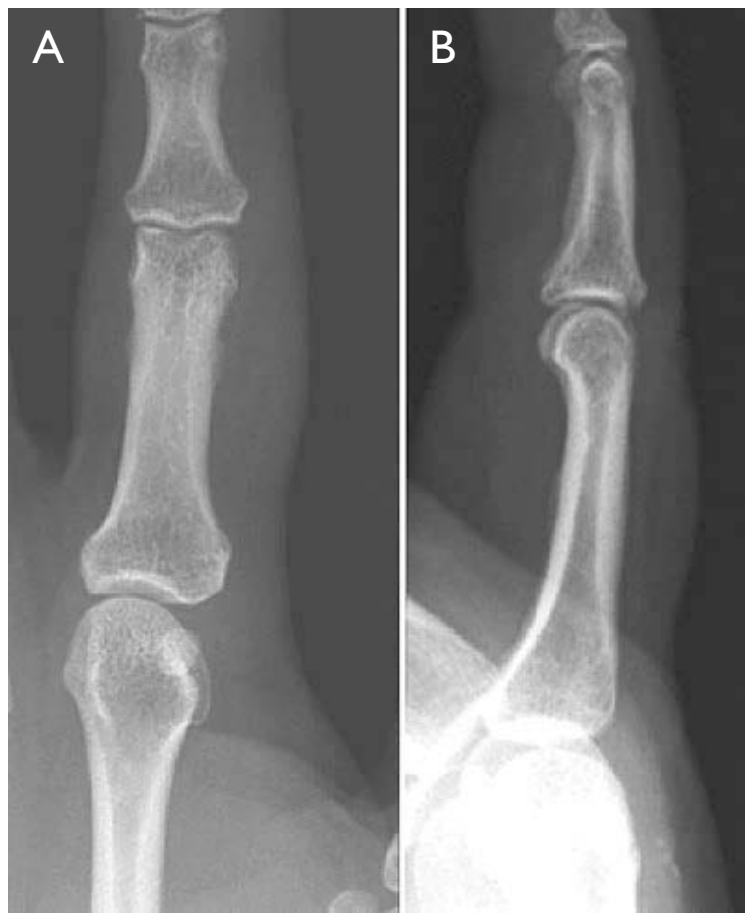

Figura. 2. Estudio radiográfico del mismo paciente al año de iniciarse la sintomatología. Obsérvese la ausencia de calcificaciones. A. Proyección anteroposterior. B. Proyección lateral.

En cuanto a la edad de aparición, suele observarse entre la segunda y quinta décadas de la vida, aunque han sido publicados algunos casos en niños ${ }^{10,1 !}$. Por lo que se refiere al género, es más frecuente en mujeres, si bien hay autores que no observan predilección por uno u otro.

El diagnóstico clínico es parecido al que acontece en las tenosinovitis agudas, motivo por el cual se las confunde con las de tipo séptico, gotoso o por depósito de pirofosfato cálcico. No obstante, el estudio sanguíneo no revela ningún parámetro relevante, aunque en algunos casos se ha observado una elevación de la VSG, tal como así fue en nuestro paciente. Es el examen radiográfico el que nos va a dar el diagnóstico definitivo. De hecho, la aparición de una calcificación, más o menos irregular y en posición para-articular, constituye un signo casi patognomónico de la entidad, al que se le dio el nombre inicial de tendinitis calcificante pero que también se le ha venido en llamar peritendinitis o periartritis calcificante, por cuanto nunca se han detectado depósitos cálcicos dentro de la sustancia tendinosa'. Por nuestra parte, la hemos denominado calcificación idiopática digital, lo que elimina el conflicto en relación a su ubicación y a su verdadera etiopatogenia. 
Finalmente y en cuanto al tratamiento se refiere, hay que decir que se trata de una patología autolimitante en el tiempo, pues su evolución natural es hacia su resolución espontánea en unas cuantas semanas. No obstante, el dolor y la inflamación que producen hacen necesaria una actuación terapéutica. En este sentido, dos son las opciones de tratamiento: la infiltración con corticoides y la administración oral de AINES/corticoides, asociadas ambas a la inmovilización del dedo afecto. La primera opción, es decir, la infiltración con corticoides, estaría reservada en los estadios iniciales, mientras que la segunda, la administración de AINE/ corticoides, estaría indicada cuando el diagnóstico se demora unos cuantos días. La indicación de cirugía sería para aquellos casos en los que los depósitos cálcicos afectaran la muñeca, especialmente los localizados dentro del canal carpiano y que se acompañan de un cuadro clínico neurológico compresivo, ya sea del nervio mediano o del nervio cubital.

\section{CONFLICTOS DE INTERESES}

Los autores declaran no tener conflictos de intereses.

\section{BIBLIOGRAFÍA}

1. McAuliffe JA. Tendon disorders of tha hands and wrist. J Hand Surg. Am. 20 I0;35A:846-53.
2. Shields JS, Chhabra AB, Pannunzio ME. Acute calcific tendinitis of the hand: 2 case reports involving the abductor pollicis brevis. Am J Orthop. 2007;36:6057.

3. Foose TE, Simon AE, Strauch RJ. Acute calcific deposition adjacent to the metacarpal head: Report or two cases and review of the literature. Orthopedics. 2005;28 (8):798-800.

4. Maseritz $\Vdash$. Acute intermetacarpophalangeal calcification. J Bone Joint Surg. 1935; 17:1017-20.

5. Cooper W. Calcareous tendinitis in the metacarpophalangeal region. J Bone Joint Surg. 1942;24: | | 4-22.

6. Seidenstein $H$. Acute pain in the wrist and hand associated with calcific deposits. J Bone Joint Surg. Am. 1950;32A:413-8.

7. Pollen AG. Calcareus deposits around the metacarpophalangeal joints. J Bone Joint Surg. Br. 1961; 43B:250-5.

8. Didley DE, Tonkin MA. Acute calcific tendonitis in the hand and wrist. J Hand Surg. Br. 1991; I 6B:21 5-6.

9. Ali AN, Kelly JL. Acute calcific tendinitis of the fingers - a case report. Hand Surg. 2004;9: 105-7.

10. Carroll RE, Seitz WH, Putnam MD. Acute calcium deposit in the hand of an I I-year-old girl. J Pediatr Orthop. 1985;5:468-70.

I I. Hakozaki M, Iwabuchi M, Konno S, Kikuchi S. Acute calcific tendinitis of the thumb in a child: a case report. Clin Rheumatol. 2007;26: 84 I-4. 